\section{Liposarcomatous differentiation in diffuse pleural mesothelioma}

\author{
J Krishna, M T Haqqani
}

\begin{abstract}
A case history is presented of a woman who died eight hours after hospital admission with severe breathlessness. At necropsy the right lung was encased in a thickened pleura with a large tumour. Histological examination of the tumour showed pleural mesothelioma with liposarcomatous differentiation. The lungs showed changes of asbestosis and the asbestos fibre count was significantly raised. Liposarcomatous differentiation in pleural mesothelioma has not been reported previously.
\end{abstract}

(Thorax 1993;48:409-410)

Three main types of malignant mesothelioma are recognised; epithelial, sarcomatoid, and mixed or biphasic types. Sarcomatoid mesothelioma with foci of osseous and cartilaginous differentiation has previously been reported. ${ }^{1}$ We report a case of diffuse malignant mesothelioma of the pleura with liposarcomatous differentiation in a patient with asbestosis and a very high fibre count, and have argued against the alternative possibility of two primary separate neoplasms.

\section{Case report}

A 77 year old woman presented with a two week history of breathlessness. A chest radiograph revealed cardiomegaly, bilateral pleural effusions, and tracheal shift to the left, suggesting a right apical mass. She had worked in a chemical packing factory for 20 years in her youth. She died eight hours after admission, before a diagnosis could be achieved.

\section{NECROPSY FINDINGS}

Pleural effusions measuring approximately $250 \mathrm{ml}$ were present on each side. The right lung was encased in a thickened pleura measuring $0.6 \mathrm{~cm}$ at the base and $2.0 \mathrm{~cm}$ at the apex, which was continuous with a fleshy large tumour with a mucoid cut surface in the upper lobe extending upwards to form a tumour mass measuring $7 \times 5.5 \times 3 \mathrm{~cm}$. The left lung showed a puckered firm area on the posterior wall of the lower border of the upper lobe measuring $2.5 \mathrm{~cm}$ in diameter, and a slightly thickened pleura measuring up to $0 \cdot 2 \mathrm{~cm}$. A greyish white tumour with a mucoid cut surface measuring $2.5 \times 1.5 \times 1$ $\mathrm{cm}$ was present in the left lower lobe reaching to within $0.1 \mathrm{~cm}$ of the pleural surface. Both lungs showed fibrosis, subpleural honeycomb changes, chronic bronchitis, and foci of bronchopneumonia. The heart was slightly enlarged (weight $430 \mathrm{~g}$, right ventricle $120 \mathrm{~g}$, left ventricle $230 \mathrm{~g}$ ). The right side was dilated and the pulmonary trunk was occluded by a large embolus. The left atrium was occupied by a large myxoid tumour mass measuring $15 \times 6 \mathrm{~cm}$ attached to a blood clot which extended into the pulmonary veins.

The rest of the organs showed no abnormalities.

\section{MICROSCOPICAL APPEARANCES}

The right pleura was diffusely infiltrated by the tumour, which showed a sarcomatous pattern with myxoid change and abundant typical lipoblasts containing sharply defined cytoplasmic vacuoles indenting hyperchromatic nuclei in the right upper lobe. This was confirmed on the electron microscope after the tissue was post fixed in osmium tetraoxide (fig 1). The right upper lobe also showed an adjacent mesothelioma with an epithelial glandular component (fig 2), uniformly negative for carcinoembryonic antigen but strongly positive for epithelial membrane antigen and cytokeratin. The liposarcomatous area was rich in vimentin and was separated from the epithelial area by thin fibrous septa. Both components were continuous with the thickened pleura. There were foci of calcification and occasional ossification in the thickened and fibrotic pleura. The tumour in the left lung was confined to the lower lobe and consisted entirely of liposarcoma. The puckered area showed fibrosis and calcification. In both lungs tumour emboli composed entirely of the liposarcomatous component were present in small and medium sized blood vessels. Both lungs also showed changes fairly typical of asbestosis-that is, diffuse fibrosis accompanied by interstitial pneumonitis, subpleural honeycomb changes, cuboidal metaplasia of the bronchial lining, and numerous asbestos bodies in both lungs, frequently forming small clusters. These were also seen diffusely

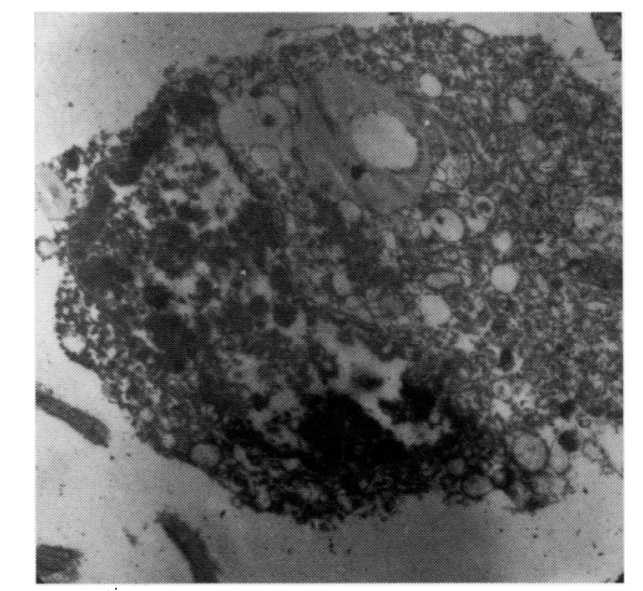

Figure 1 Electron microscopic appearance of a lipoblast showing indentation of the nucleus and cytoplasmic vacuoles. 


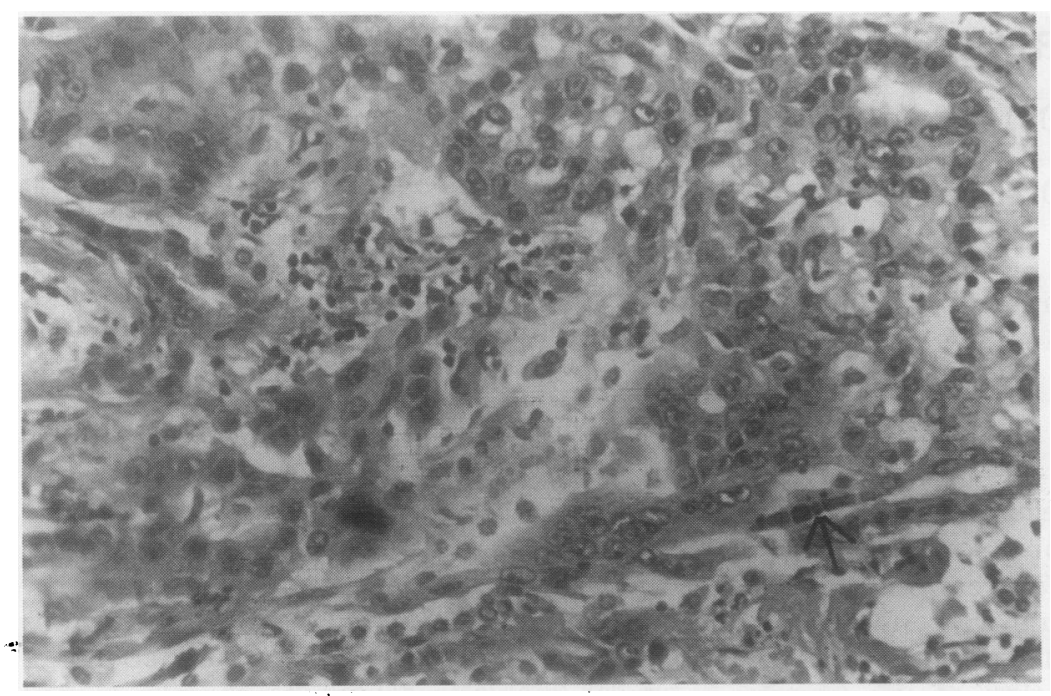

Figure 2 Malignant epithelial area with irregular glands lined by pleomorphic cuboidal epithelium. An asbestos body is marked by an arrow in the right hand corner (haematoxylin and eosin). in both tumour components within the glandular spaces or interstitial tissue, or occasionally in the cytoplasm of the lipoblasts.

The asbestos fibre count/g dried lung, as determined by the method of Ashcroft and Hepplestone, ${ }^{2}$ was in excess of 11 million fibres (normal fibre count $30000-35000$ ).

The tumour occupying the left atrium was entirely composed of myxoid liposarcoma spreading via the pulmonary veins.

\section{Discussion}

The two entirely different tumour patterns described in this case may either be liposarcomatous differentiation in diffuse pleural mesothelioma, or two separate primary malignant tumours. We favour the former possibility for the following reasons.

The mesothelial cell is pluripotent since it originates from the mesoderm which is formed from both ectodermal and endodermal layers. ${ }^{3}$ Malignant mesothelioma is thought to arise from a primitive submesothelial precursor of mesothelial cells or the mesothelial cells themselves. ${ }^{4}$ Donna and Betta reviewed biopsy and necropsy specimens from mesothelial tumours and described myoblastic, angioblastic, lymphoblastic, chondroblastic, and fibroblastic differentiation. They suggested the concept of "mesodermoma" to explain the varied forms of differentiation. ${ }^{5}$ Other case reports of tumours in asbestos workers arising from the pleura or the chest wall have described bone and cartilage formation. ${ }^{16}$ Primary liposarcoma of the pleura without mesothelioma has also been reported. ${ }^{7}$ The tumour described in this report shows widely scattered asbestos bodies in both tumour components. Only one other case report has described asbestos bodies in a sarcomatoid mesothelioma of the chest wall, ${ }^{6}$ and in their paper the authors stated that the presence of asbestos bodies was important in establishing the cause of sarcomatoid mesothelioma. Since there was no intermingling of liposarcomatous and epithelial components in our case, two primary malignant tumours cannot entirely be excluded. We believe, however, that the tumour presented in this report is a liposarcomatous differentiation in a diffuse mesothelioma. This assertion is also supported by the observation that mesotheliomas of sarcomatous pattern can show many sorts of differentiation, bone and cartilage being particularly well recognised. Any diffuse sarcoma of the pleura is best regarded as a mesothelioma, but liposarcomatous differentiation in mesothelioma has not been observed previously (B Corrin, personal communication).

We wish to thank Professor B Corrin, Professor of Thoracic Patholosy Brompton Hospital, for a review of the slides and permission to quote his personal observations, and Mrs B Nettleton for typing the manuscript. We wish also to thank $W$ Fellows for help with electron microscopy.

1 Yousem SA, Hochholzer L. Malignant mesotheliomas with osseous and cartilaginous differentiation. Arch Pathol Lab Med 1987;111:62-6.

2 Ashcroft T, Heppleston AG. The optical and electron microscope determination of pulmonary asbestos fibre concentration and its relation to human pathology. $\mathcal{F}$ Clin Pathol 1973;26:224-34.

3 Donna A, Provana A. Considerations and proposals about mesotheliomas based on morphologic appearances. I. Histological consideration in optic microscopy. Pathologica 1977;69:441-68.

4 Hillerdal G. Malignant mesotheliomas 1982: review of 4710 published cases. $B r \mathcal{F}$ Dis Chest 1983;77:321-43.

5 Donna A, Betta PG. Mesodermoma: new embryological approach to primary tumours of coelomic surfaces. Histopathology 1981;5:31-44.

6 Andrion A, Mazzucco G, Bernardi P, Mollo F. Sarcomatous tumour of the chest wall with osteochondroid differentiation. Evidence of mesothelial origin. Am f Surg Pathol 1989;13:707-12.

7 Evans AR, Wolstenholme RJ, Shettar SP, Yogish H. Primary pleural liposarcoma. Thorax 1985;40:554-5. 